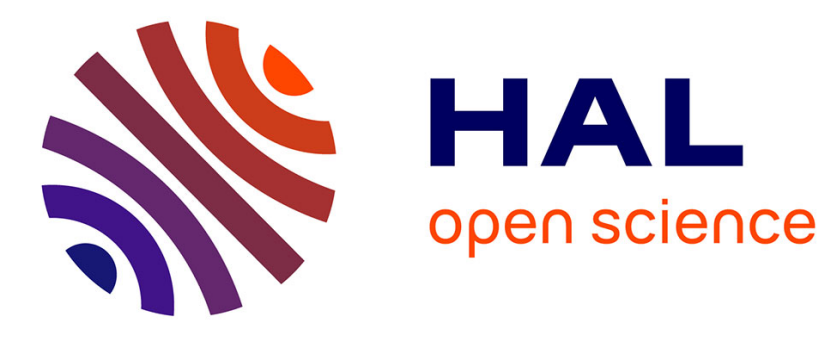

\title{
Modelling of high frequency vibro-impact drilling
}

\author{
Ekaterina Pavlovskaia, David C. Hendry, Marian Wiercigroch
}

\section{To cite this version:}

Ekaterina Pavlovskaia, David C. Hendry, Marian Wiercigroch. Modelling of high frequency vibro-impact drilling. International Journal of Mechanical Sciences, 2015, 91, pp.110 - 119. 10.1016/j.ijmecsci.2013.08.009 . hal-01634311

\section{HAL Id: hal-01634311 https://hal.science/hal-01634311}

Submitted on 13 Nov 2017

HAL is a multi-disciplinary open access archive for the deposit and dissemination of scientific research documents, whether they are published or not. The documents may come from teaching and research institutions in France or abroad, or from public or private research centers.
L'archive ouverte pluridisciplinaire HAL, est destinée au dépôt et à la diffusion de documents scientifiques de niveau recherche, publiés ou non, émanant des établissements d'enseignement et de recherche français ou étrangers, des laboratoires publics ou privés. 


\title{
Modelling of high frequency vibro-impact drilling
}

\author{
Ekaterina Pavlovskaia*, David C. Hendry, Marian Wiercigroch \\ Centre for Applied Dynamics Research, School of Engineering, King's College, Aberdeen University, Aberdeen AB24 $3 U E$, UK
}

\begin{abstract}
Modelling of the vibro-impact drilling system is undertaken in this study, and the results of the numerical analysis and comparison between two selected models are presented. The first one is a newly developed model of an existing experimental rig (three masses model) and the second one is a simplified low dimensional model (Pavlovskaia et al., 2001 [22]) created to describe the dynamic interaction between the drill-bit and the drilled formation. The optimal loading parameters are identified in this work based on the analysis of the system responses, and the influence of the additional degrees-offreedom on the loading optimisation strategy is investigated. Three main control parameters are considered, and they are the applied static force, and the amplitude and the frequency of the applied dynamic force.

Our investigations confirm that the best progression rates are achieved when the system response is periodic and the frequency of the response is the same as the frequency of the applied dynamic force, and the value of the static force is smaller than the amplitude of the applied dynamic excitation. This result is valid for both models considered. Also in both cases, zero progression rates are obtained for lower values of the excitation amplitudes and the average progression increases with the increase in the dynamic amplitudes. Both models also predict zero progression rates at the higher excitation frequencies.

Based on the analysis undertaken, it can be concluded that the low dimensional model provides good estimates of the optimal static force and the amplitude of the dynamic force, and it could be used for the operational control of the drilling system to adjust the loading parameters while drilling through different formations. The choice of the optimal frequency, however, should be made based on the predictions of the more detailed model of the drilling system as additional degrees of freedom significantly influence the structure of the internal resonances and they should be taken into account.
\end{abstract}

\author{
Keywords: \\ Vibro-impact drilling \\ Modelling \\ Optimal progression
}

\section{Introduction}

The idea of utilising impact energy to drill rock formations has been tried by the engineering community since the late 1940s, when a number of tools were developed known as downhole hammers, percussive hammers, percussive drills and others [1]. They all had a similar operating principle, where a compressed-air or hydraulically operated piston impacts upon a drilling rod (or series of rods) transferring the potential energy into kinetic energy of the drill-bit [2]. As a result, rocks are chipped and crushed and the drill-bit together with the drill-string penetrate into the rock. To further enhance penetration rates, rotary action may be superimposed upon the percussive motion of the drill bit. A fresh area of the rock surface is then presented to the drill-bit at each successive blow provided that cuttings are being effectively removed from the drilling zone.

\footnotetext{
* Corresponding author. Tel.: +44 1224272786.

E-mail address: e.pavlovskaia@abdn.ac.uk (E. Pavlovskaia).
}

The performance of percussive hammers [1] has been studied extensively both in the laboratory and in the field. Guarin et al. [3] presented one of the first reports on the usage of the rotary percussion-drilling technique to drill an almost $4 \mathrm{~km}$ deep well. The authors considered the effect of weight on bit (WOB) and rotary speed upon the tool performance in various formations, and also studied the behaviour of the various drill-bits during percussive drilling. In general, substantial improvements in penetration rates were achieved in comparison with rotary drilling. In 1958 Topanelian [4] conducted an experimental study in the laboratory investigating the effect of the frequency of percussive drilling in the range from 5 to $17 \mathrm{~Hz}$ (low frequency range) on granite blocks. In these experiments the drill-bit was held stationary and the test block was rotated and forced upwards by a hydraulically lifted rotary table. It was concluded that using percussive action more than doubled the penetration rate normally produced by the static WOB. In 1964 Bates [5] presented some field results of percussive air drilling. He has demonstrated that the pressure to operate the percussion tool was the most significant single factor affecting the progression and that selection of the bit was very important for 
the operation. Further field results were presented by Smith and Kopczynski [6] who also concluded that "air-rotary-percussive drilling is a valuable tool for the industry" and requires further development and optimisation.

The fundamental mechanisms of percussive drilling were investigated by a number of researchers including Simon [7,8], Hustrulid and Fairhurst [9-12], Lundberg [13-17], Franca and Weber [18,19], and the Centre for Applied Dynamics Research in Aberdeen [20-26]. Simon $[7,8]$ considered the energy in the stress wave in the drill steel produced by the striker impact together with the energy required for breaking the rock and computed the efficiency of stress wave energy conversion into work done by the bit on the rock. He also discussed the possibilities of improving utilisation of the energy to drill rock. Hustrulid and Fairhurst analysed the efficiency of impact energy transfer from drill to rock both theoretically [9] and experimentally [11]. They also studied the forcepenetration relationship both in static and dynamic loading conditions using indentation tests and a "drop test" [10]. In [12] a series of full-time drilling tests made using three different percussive machines were presented, where blow energy, blow frequency, rotational speed, thrust, penetration rate, and the impact-produced strain waves were measured. It was shown that the minimum thrust required for optimum energy transfer to the rock is a function of the blow frequency and the initial and rebound momentum of the piston, the latter depending on the incident waveform and the force-penetration curve. Aspects of energy conversion, energy transfer and efficiency were considered in [13-17] by Lundberg and his co-authors, who also compared the efficiencies of different percussive processes in these papers. With the exception of [16], where dynamic 3D models were used, wave phenomena in drills have generally been studied using 1D wave theory.

Drifting oscillator models suitable for percussive drilling dynamics description were introduced in $[20,22]$ and have been extensively studied in the past, see for example [23,24], to find the optimum characteristics of the applied static and dynamic forces. In these studies the impacted media was represented by the socalled sliders which provided the contact force acting on the drillbit during the interactions. These models were developed further in $[25,26]$ to take into account the influence of contact geometries and the governing force-displacement relationship during the crashing stages of the interactions.

Franca [18] conducted a series of experiments on an in-house designed rotary-percussive drilling rig and proposed a phenomenological bit-rock interaction model for rotary-percussive drilling aiming to obtain quantitative information from drilling data related to rock properties, bit conditions and drilling efficiency. More recently Franca and Weber [19] conducted experimental and numerical studies of a new resonance hammer drilling model with drift and showed that the behaviour of the system may vary significantly from simple periodic regimes to chaos. Wiercigroch et al. [21] presented extensive studies of ultrasonic percussive drilling with diamond-coated tools in the laboratory conditions on rocks such as sandstone, limestone, granite and basalt. The studies aimed to explore the applicability of this technique to downhole drilling and were supported by the development of mathematical models capable of describing the main phenomena occurring during drilling.

Despite these technological advances, significant scientific interest and activities in this area, there are still a number of challenges to be addressed for the technology to become the norm in the industry $[1,27]$. An extensive research programme conducted in the University of Aberdeen in the last few years aims to develop the Resonance Enhanced Drilling (RED) technique [28] and this paper will present some of the results of the mathematical modelling and analysis obtained during this project. The main idea behind this technology is to apply an adjustable high frequency dynamic stress (generated by axial oscillations) in combination with rotary action in order to enhance the penetration rates by creating resonance conditions between the drill-bit and the drilled formation. This resonance needs to be maintained for varying drilling conditions by adjusting the frequency and amplitude to produce a steadily propagating fracture zone and it is particularly beneficial while drilling the hard rocks.

An experimental rig shown in Fig. 1 was designed and manufactured to explore these ideas. To assist data gathering during experiments, in the current rig the vertical oscillations of the drillbit generated by a PEX magneto-strictive device and static weight on bit provided by the hydraulic cylinder are separated from the rotary motion of the specimen which is supplied by the rotating table of the vertical lathe. This arrangement allows for a significant simplification of the rig instrumentation but maintains the combined rotary percussive action applied at the rock/drill-bit interface.

This paper is focussed on the modelling of the percussive component of the system dynamics and it presents the results of the numerical analysis and a comparison of the two models. The first one is a newly developed model of the experimental rig and the second one is a simplified low dimensional model [22] created to describe the dynamic interaction between the drill-bit and the drilled formation. Our aim is to identify how the complexity of the model including additional degrees of freedom influences the prediction of the optimal loading parameters. The rest of the paper is organised as follows. In Section 2 the mathematical model of the experimental rig is introduced and the equations of motion are presented. The next section describes typical behaviour of the system and examines the influence of the external force parameters on the system dynamics. Section 4 presents the comparison between the predictions of the optimal loading calculated according to this new model and the model introduced in [22].

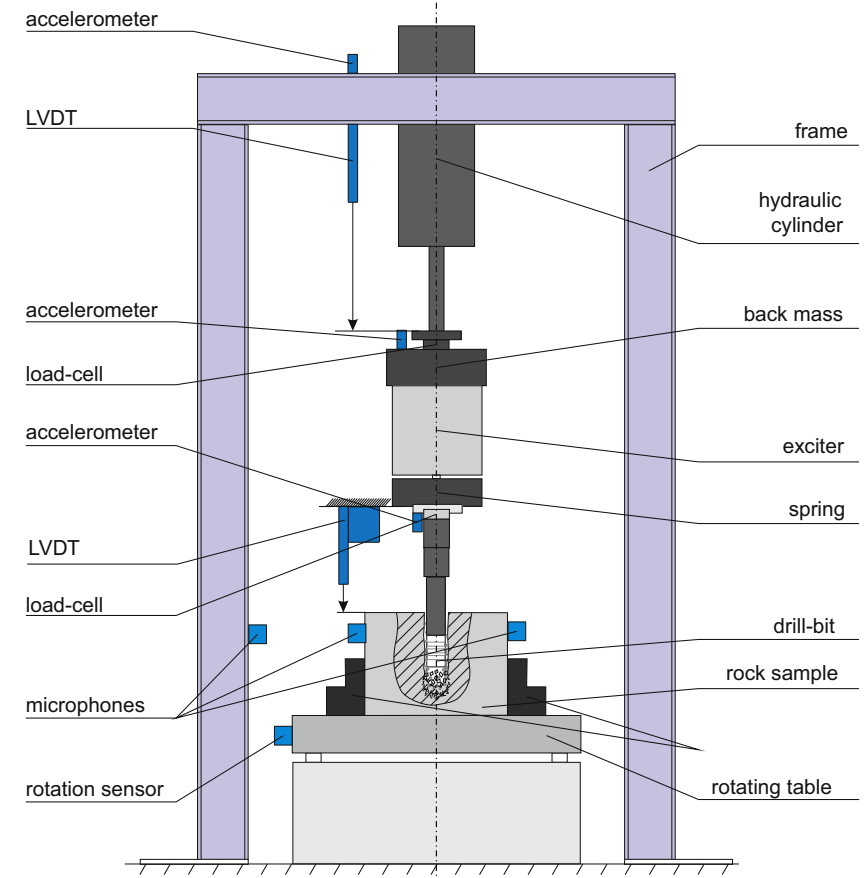

Fig. 1. Schematic of the experimental rig. The drill string consists of a number of elements including exciter (PEX magneto-strictive device) providing the dynamic external force, two structural springs and back mass to control vibrations within the system, and a drill-bit. The static force is applied using the hydraulic cylinder which drives the system downwards when the rock is broken and the progression takes place. The rotary action is generated through the rotating table where only the rock specimen is rotated. As indicated there are a number of load-cells, LVDTs and other sensors to monitor the system dynamics. 


\section{Physical and mathematical modelling}

The RED drill-string is modelled as a three mass system as shown in Fig. 2, where a frictional slider has been included to account for the rock dynamics. This model facilitates an insight into the overall dynamics of the RED drill-string during drilling operations.

The drill-bit is modelled by a mass $m_{1}$, which is initially positioned at a distance $G$ from the surface of the rock. The rock is modelled by a visco-elastic slider [22]. For small contact forces, the spring of stiffness $k_{1}$ and damper of damping coefficient $c_{1}$ account for the elastic behaviour and the damping of the rock. However, if the force acting on the slider would exceed $P_{r}$, it starts to move while maintaining a constant resistive force $P_{r}$. Here, the displacement of the drill-head is $x_{1}$, that of the rock surface is $x_{t}$ and the displacement of the slider is $x_{b}$. When the drill-head and the rock are not in contact, the velocity of the slider is zero and provides no force, i.e. $P_{c}=0$. During this free motion regime the surface of the rock moves $\left(x_{t}\right)$ under the forces of the spring $k_{1}$ and the damper $c_{1}$. When the drill-head is in contact with the rock, the displacement $x_{1}$ of the drill-head and $x_{t}$ of the rock surface differ by the value of initial gap $G$ only.

The equivalent oscillating mass of the frame is modelled as the top mass $m_{3}$. This is a large mass when compared to the two others connected to the mass of the exciter $m_{2}$ by a spring of constant $k_{3}$ and a damping coefficient $c_{3}$. The resulting equation of motion for this mass is therefore given as

$m_{3} \ddot{x}_{3}+c_{3}\left(\dot{x}_{3}-\dot{x}_{2}\right)+k_{3}\left(x_{3}-x_{2}\right)=0$.

The mass of the RED module is marked as $m_{2}$ and it is connected to the drill-bit via a structural spring that has a spring constant of $k_{2}$ and a damping coefficient of $c_{2}$. The resulting equation of motion for this part of the model is given by

$m_{2} \ddot{x}_{2}+c_{3}\left(\dot{x}_{2}-\dot{x}_{3}\right)+c_{2}\left(\dot{x}_{2}-\dot{x}_{1}\right)+k_{3}\left(x_{2}-x_{3}\right)+k_{2}\left(x_{2}-x_{1}\right)=F_{s}$.

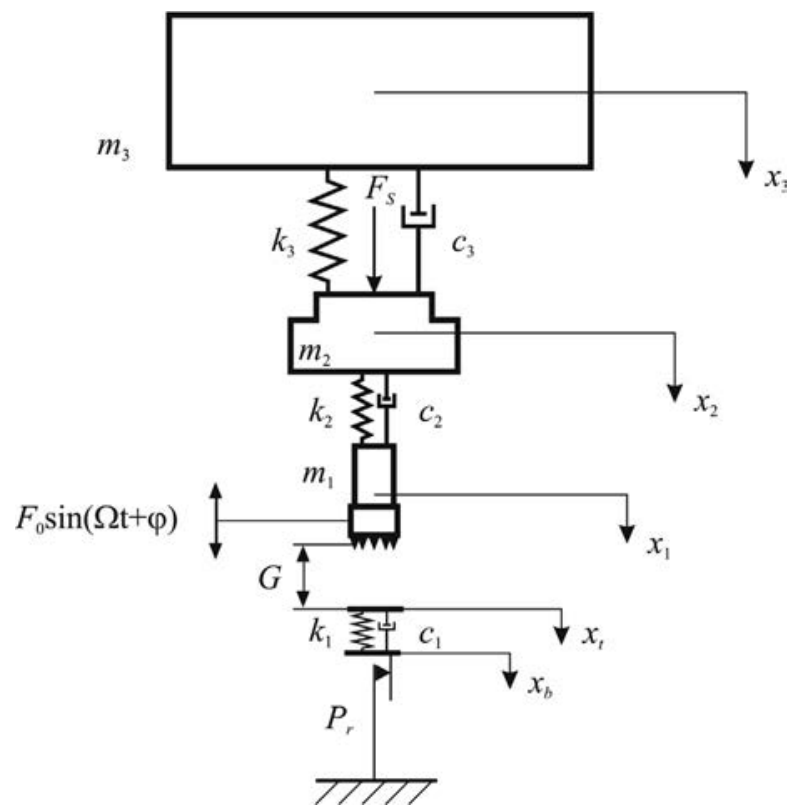

Fig. 2. Physical model of the experimental rig. Here the drill-bit is modelled by mass $m_{1}$, mass $m_{2}$ represents the RED module and mass $m_{3}$ accounts for the equivalent oscillating mass of the frame. The rock is modelled by a visco-elastic slider [22], where the elastic behaviour and the damping of the rock are described by the spring of stiffness $k_{1}$ and damper of damping coefficient $c_{1}$. When the force acting on the slider exceeds $P_{r}$, the whole system moves downward simulating the progression of the drill-bit.
The bottom mass $m_{1}$ is the drill bit and it has three different regimes of operation depending on its position with respect to the rock surface.

No contact:

$x_{1}<x_{t}+G$

This first stage, No contact, lasts until the drill bit reaches the slider at $x_{1}=x_{t}+G$. During this stage the contact force is $P_{c}=k_{1}\left(x_{t}-x_{b}\right)+c_{1}\left(\dot{x}_{t}-\dot{x}_{b}\right)=0$, and the mass, the rock surface and the slider move according to the following equations of motion:

$m_{1} \ddot{x}_{1}+k_{2}\left(x_{1}-x_{2}\right)+c_{2}\left(\dot{x}_{1}-\dot{x}_{2}\right)=F_{0} \cos (\Omega t+\phi)$,

$\dot{x}_{t}=-\frac{k_{1}}{c_{1}}\left(x_{t}-x_{b}\right)$,

$\dot{x}_{b}=0$.

Contact without progression:

$x_{1} \geq x_{t}+G, \quad \dot{x}_{1}>0, \quad 0<k_{1}\left(x_{t}-x_{b}\right)+c_{1}\left(\dot{x}_{t}-\dot{x}_{b}\right)<P_{r}$

The contact force can be computed as

$P_{c}=k_{1}\left(x_{t}-x_{b}\right)+c_{1}\left(\dot{x}_{t}-\dot{x}_{b}\right)$.

This stage ends when the contact force $P_{c}$ exceeds the threshold $P_{r}$, then the third stage starts (see below). If the contact force $P_{c}$ falls below zero, contact stops, and stage 1 starts (see above). During this stage the massless rock surface moves together with the drill bit, $x_{t}=x_{1}-G, \dot{x}_{t}=\dot{x}_{1}$ and $\dot{x}_{b}=0$. The resulting equations of motion for this phase are given by

$m_{1} \ddot{x}_{1}+c_{2}\left(\dot{x}_{1}-\dot{x}_{2}\right)+c_{1} \dot{x}_{1}+k_{2}\left(x_{1}-x_{2}\right)-k_{1}\left(G+x_{b}\right)=F_{0} \cos (\Omega t+\phi)$, $x_{t}=x_{1}-G$,

$\dot{x}_{b}=0$.

Contact with progression:

$x_{1} \geq x_{t}+G, \quad \dot{x}_{1}>0, \quad k_{1}\left(x_{t}-x_{b}\right)+c_{1}\left(\dot{x}_{t}-\dot{x}_{b}\right) \geq P_{r}$.

In this case the slider moves under the combined action of the resistance force $P_{r}$ and the spring between the slider and the rock surface, and due to its zero mass we have

$k_{1}\left(x_{t}-x_{b}\right)+c_{1}\left(\dot{x}_{t}-\dot{x}_{b}\right)-P_{r}=0$.

The contact force acting on the drill-bit in this case is equal to the resistance force $P_{r}$. Since the drill-bit moves together with the rock surface, $\dot{x}_{t}=\dot{x}_{1}$, the equation for the motion of the slider can be expressed from the above equation as

$\dot{x}_{b}=\dot{x}_{t}+\frac{k_{1}}{c_{1}}\left(x_{t}-x_{b}\right)-\frac{P_{r}}{c_{1}}$.

This stage lasts until the value of $k_{1}\left(x_{t}-x_{b}\right)+c_{1}\left(\dot{x}_{t}-\dot{x}_{b}\right)$ falls below $P_{r}$, then stage 2 above restarts. The resulting equations of motion for this phase are given by

$$
\begin{aligned}
& m_{1} \ddot{x}_{1}+c_{2}\left(\dot{x}_{1}-\dot{x}_{2}\right)-c_{1}\left(\dot{x}_{1}-\dot{x}_{b}\right)+k_{2}\left(x_{1}-x_{2}\right)-k_{1}\left(x_{t}-x_{b}\right) \\
& \quad=F_{0} \cos (\Omega t+\phi), \\
& x_{t}=x_{1}-G, c_{1}\left(\dot{x}_{1}-\dot{x}_{b}\right)+k_{1}\left(x_{1}-G-x_{b}\right)=P_{r} .
\end{aligned}
$$

To make the simulations clearer, the equations of motion are transformed into a non-dimensional form. The non-dimensional parameters and variables are derived as follows:

$\Omega_{0}=\sqrt{\frac{k_{1}}{m_{1}}}, \quad \omega=\frac{\Omega}{\Omega_{0}}, \quad a=\frac{F_{0}}{P_{r}}$,

$b=\frac{F_{s}}{P_{r}}, \quad \xi=\frac{c_{1}}{2 m_{1} \Omega_{0}}, \quad g=\frac{k}{P_{r}} G$,

$\alpha_{3}=\frac{m_{1}}{m_{3}}, \quad \alpha_{2}=\frac{m_{1}}{m_{2}}, \quad \beta_{3}=\frac{k_{3}}{k_{1}}$,

$\beta_{2}=\frac{k_{2}}{k_{1}}, \quad \gamma_{3}=\frac{c_{3}}{c_{1}}, \quad \gamma_{2}=\frac{c_{2}}{c_{1}}$,

$\tau=\Omega_{0} t, \quad z_{1}=x_{3} \frac{k_{1}}{P_{r}}, \quad z_{2}=\frac{d z_{1}}{d \tau}, \quad z_{3}=x_{2} \frac{k_{1}}{P_{r}}$, 
$z_{4}=\frac{d z_{3}}{d \tau}, \quad z_{5}=x_{1} \frac{k_{1}}{P_{r}}, \quad z_{6}=\frac{d z_{5}}{d \tau}$

$z_{7}=x_{t} \frac{k_{1}}{P_{r}}, \quad z_{8}=x_{b} \frac{k_{1}}{P_{r}}$.

Finally, we get the equations

$z_{1}^{\prime}=z_{2}$

$z_{2}^{\prime}=-\alpha_{3} \beta_{3} z_{1}-2 \xi \alpha_{3} \gamma_{3} z_{2}+\alpha_{3} \beta_{3} z_{3}+2 \xi \alpha_{3} \gamma_{3} z_{4}$

$z_{3}^{\prime}=z_{4}$

$z_{4}^{\prime}=\alpha_{2} \beta_{2} z_{1}+2 \xi \alpha_{2} \gamma_{2} z_{2}-\alpha_{2}\left(\beta_{2}+\beta_{3}\right) z_{3}-2 \xi \alpha_{2}\left(\gamma_{2}+\gamma_{3}\right) z_{4}+\alpha_{2} \beta_{2} z_{5}+\alpha_{2} b$

$z_{5}^{\prime}=z_{6}$

$z_{6}^{\prime}=a \cos (\omega \tau+\phi)-\beta_{2}\left(z_{5}-z_{3}\right)-2 \xi \gamma_{2}\left(z_{6}-z_{4}\right)$

$$
-P_{1} P_{2}\left(1-P_{3}\right)\left(2 \xi z_{6}+z_{7}-z_{8}\right)-P_{1} P_{3}
$$

$z_{7}^{\prime}=P_{1} z_{6}-\left(1-P_{1}\right)\left(z_{7}-z_{8}\right) /(2 \xi)$

$z_{8}^{\prime}=P_{1} P_{2} P_{3}\left(\left(z_{7}-z_{8}-1\right) /(2 \xi)+z_{6}\right)$,

where $\quad P_{1}=H\left(z_{7}-z_{8}\right), \quad P_{2}=H\left(2 \xi z_{6}+z_{7}\right), \quad P_{3}=H\left(2 \xi z_{6}+z_{7}-1\right)$, $P_{4}=H\left(z_{6}\right)$ and $H(\cdot)$ is the Heaviside step function. These ordinary differential equations were solved numerically using standard subroutine in FORTRAN based on Adams-Moulton's method and the results are presented in the following sections.

\section{Dynamics of a large system}

As it has been shown earlier [29-31], the periodic regimes are most beneficial from the practical point of view of achieving the best progression rates. It should be noted that since the system considered is piecewise linear, its dynamic response can be constructed by stitching linear solutions at points of discontinuities.
A typical sequence of period one motion is comprised of the following phases:

- Phase I: Progression; the mass and the slider are in contact.

- Phase II: Contact without progression; the mass and the slider are in contact but the slider bottom is not moving.

- Phase III: No contact; the mass and the slider are moving separately.

- Phase IV: Contact without progression; the same as Phase II.

Fig. 3 demonstrates such typical period-1 response of the system. Here and in Figs. 4 and 5, the curves related to the top mass are shown by black lines, those related to the middle (back) mass are in red and those related to the drill-bit are in blue. Parts (a)-(c) of these figures demonstrate motions of the three masses on the special phase plane where the relative displacement between the masses and the slider bottoms is shown. Part (d) presents displacements of the masses as a function of time for a chosen time interval long after the initial transition period. Finally, in part (e) the relative displacements as functions of time are shown.

As can be seen from Fig. 3(c), for the period-1 response, there is one impact per period of external excitation and the system is moving forward achieving good penetration rate. Here the mass $m_{3}$ experiences very little oscillatory motion as can be seen from its trajectory given by the black line in Fig. 3(d), and the energy in the system is focussed to produce the oscillations of the drill-bit capable of generating high impact forces to break rock and progress forward.

Two other possible system responses are shown in Figs. 4 and 5. The first one presents period-2 response observed for higher a

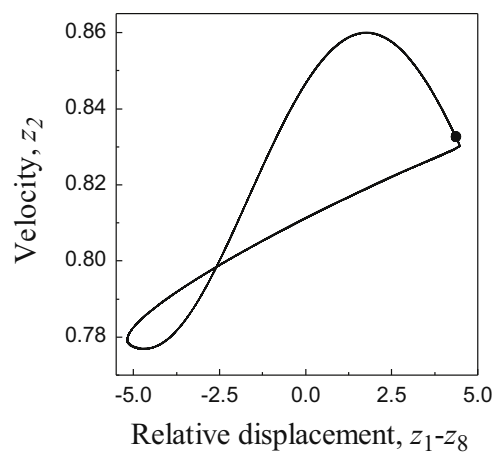

b

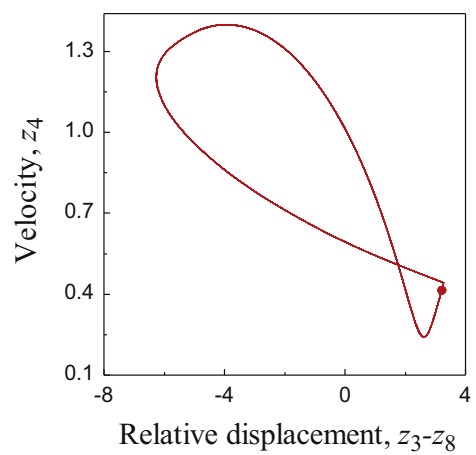

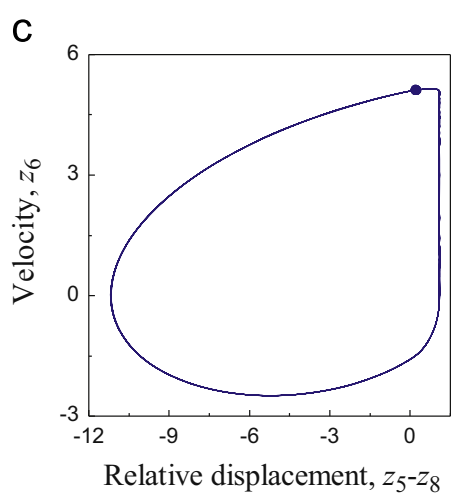

d

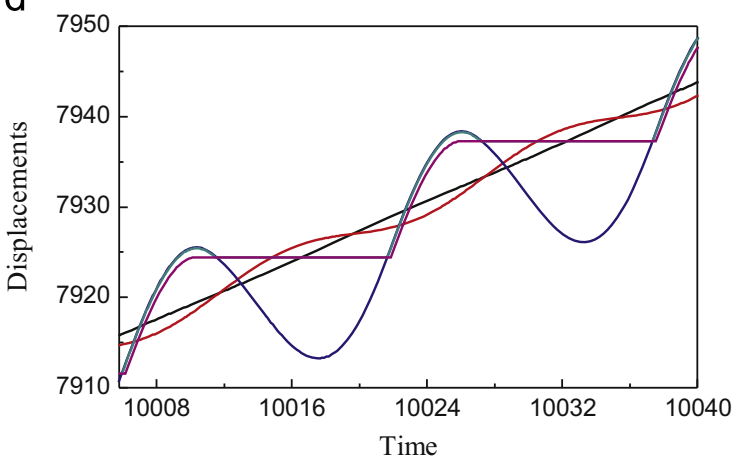

e

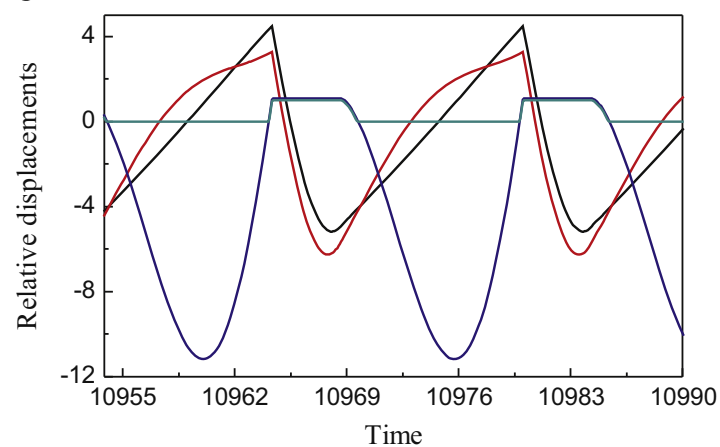

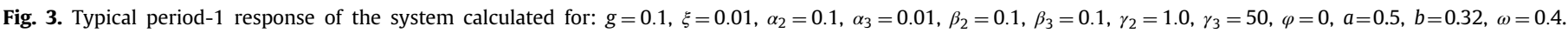

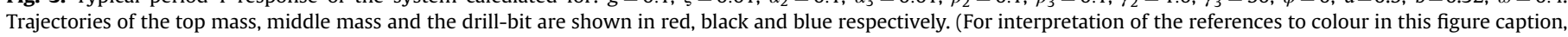
the reader is referred to the web version of this article.) 
a

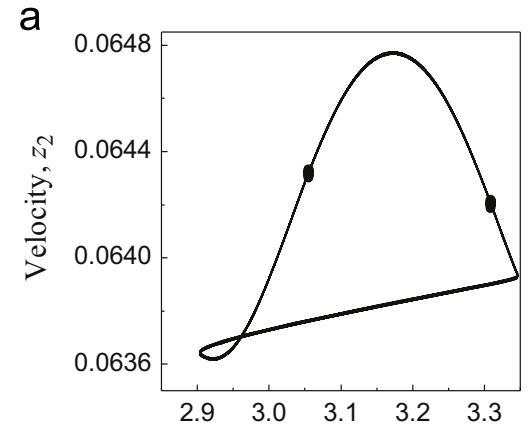

Relative Displacement, $z_{1}-z_{8}$ $\mathrm{b}$

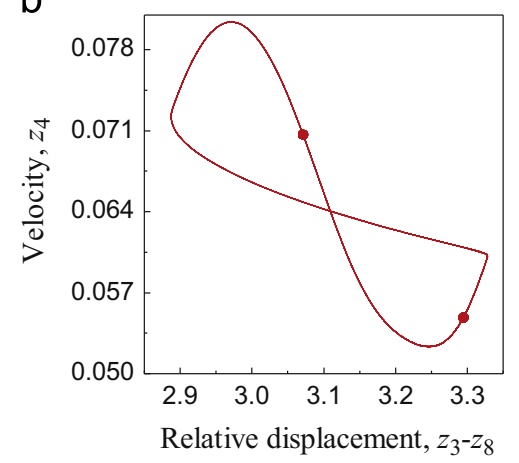

C

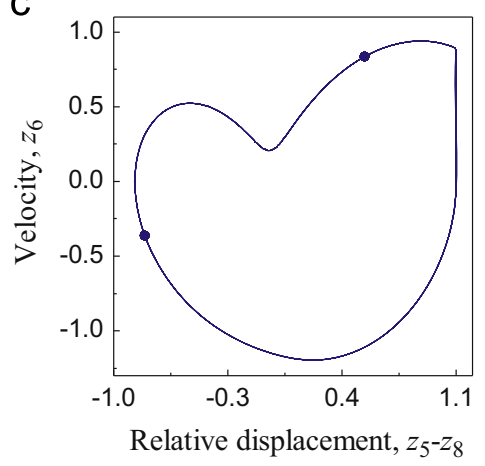

d
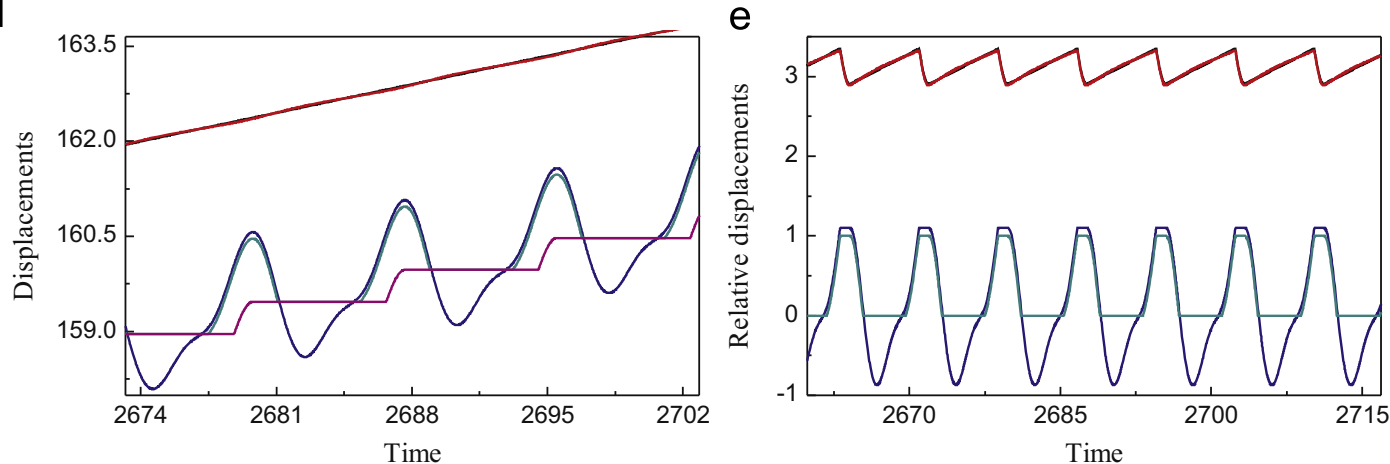

Fig. 4. Typical period-2 response of the system calculated for: $g=0.1, \xi=0.01, \alpha_{2}=0.1, \alpha_{3}=0.02, \beta_{2}=0.1, \beta_{3}=0.1, \gamma_{2}=1.0, \gamma_{3}=50, \varphi=0, a=0.7, b=0.3, \omega=1.6$. Trajectories of the top mass, middle mass and the drill-bit are shown in red, black and blue respectively. (For interpretation of the references to colour in this figure caption, the reader is referred to the web version of this article.)

a

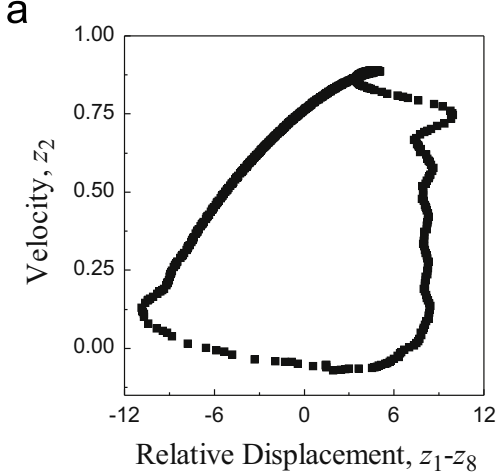

b

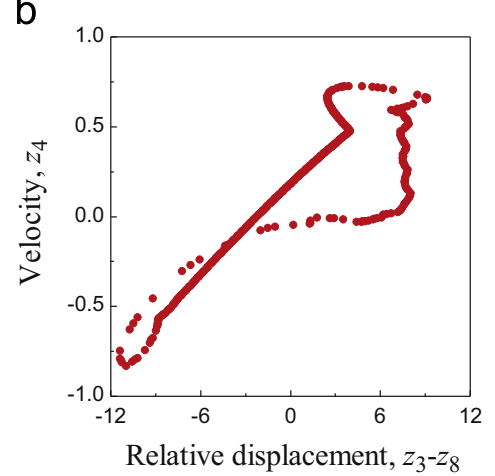

C

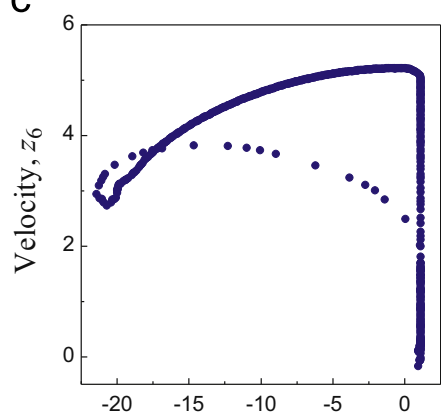

Relative displacement, $z_{5}-z_{8}$
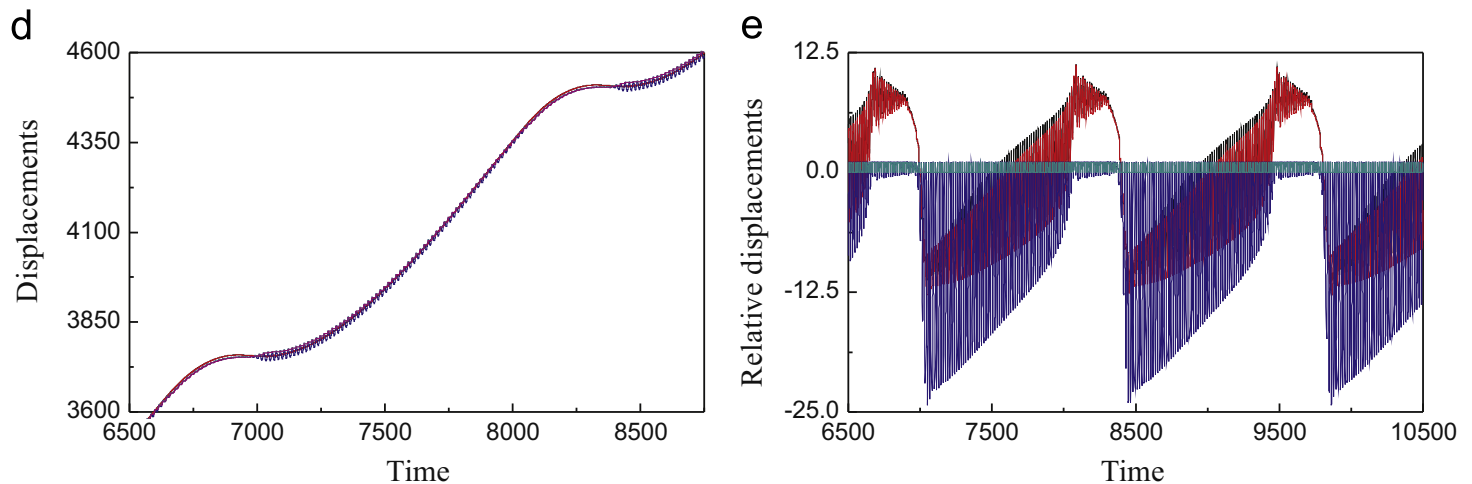

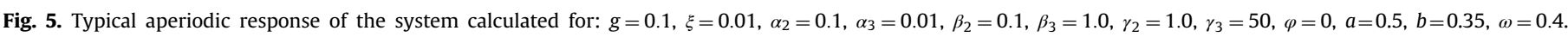

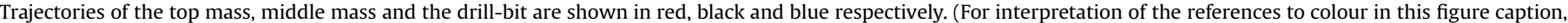
the reader is referred to the web version of this article.) 
excitation frequency but similar ratio of static and dynamic forces. Here the drill-bit experiences one impact per two periods of external excitation and the progression rate achieved is much lower. In Fig. 5 typical aperiodic response is shown. As can be seen the level of vibrations is higher in this case but the penetration rate is lower, and therefore such a combination of the excitation parameters should be avoided.

Our simulations revealed that a large variety of system responses could be obtained depending on the excitation parameters, and therefore the influence of these parameters on the dynamic behaviour of the system has been studied. The main aim of these investigations was to generate optimal parameter combinations that would lead to enhanced penetration rates in the RED test rig. The average penetration rate (or progression per period) over a significantly long period of time has been chosen to monitor the system performance. The optimal progression was sought for different system configurations by modifying three main control parameters which are the static force, the excitation frequency and the excitation amplitude. In the next subsections the results of simulations are presented where the influence of these control parameters on progression rate was considered for a selected set of the system parameters describing the experimental rig.

\subsection{Influence of static force}

The static force was considered first. It is intuitive that the static force (i.e. weight on bit) will have a strong influence on the progression achieved. Fig. 6(a) shows the trend followed by the average progression with changes in the level of the static force applied. Five different curves are presented and they are obtained for different amplitudes of the dynamic excitation amplitude, $a=F_{0} / P_{r}$.

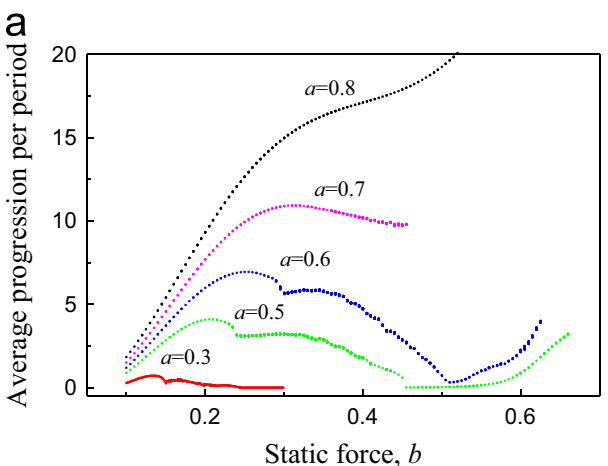

It can be seen that for every curve except for $a=0.8$, there is a well pronounced maximum up to which point the progression is smooth. Our simulations show that as soon as the static force is increased beyond this maximum, the system response becomes chaotic and the average progression per period decreases. Typical bifurcation diagram calculated for $a=0.5$ and $g=0.1, \xi=0.01$, $\alpha_{2}=0.1, \alpha_{3}=0.2, \beta_{2}=0.1, \beta_{3}=0.01, \gamma_{2}=1, \gamma_{3}=50, b=0.35$, $\varphi=0$ and showing the system response under varying static force is presented in Fig. 6(b). It can be seen that in the considered range of the static force $b$, the periodic response of the system is observed for $b \in(0.1,0.225)$, which is followed by a reasonably large window of chaotic motion for $b \in(0.225,0.450)$. The beginning of the range of chaotic behaviour corresponds to the visible drop in the average progression and by the end of this range the average progression becomes almost zero.

Similar analysis was performed for the other values of the excitation amplitude, and it was shown that for any chosen dynamic amplitude, an optimal static force can be identified from the region where the system response is periodic. As can be seen from Fig. 6(a), for the smaller values of the dynamic amplitudes $(a=0.3,0.5,0.6)$ the average progression can drop as low as zero as the static force increases. However when the static force increases further, progression per period may become larger again, but that would require a significant increase in the energy pumped into the system.

\subsection{Influence of excitation amplitude}

The amplitude of the dynamic high-frequency excitation is another major parameter that could influence the average progression. Fig. 7(a) demonstrates the dependence of average progression per period on the dynamic amplitude for three different

b

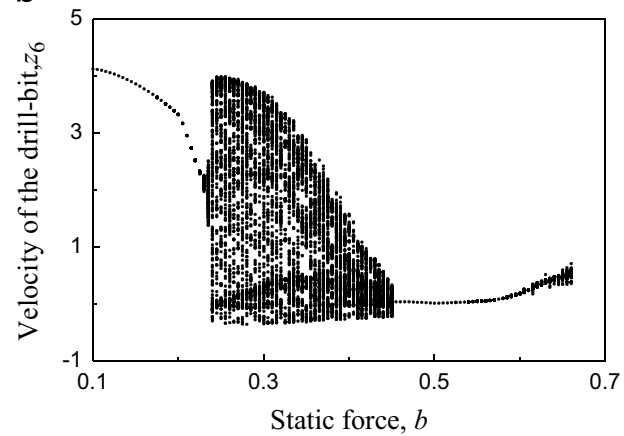

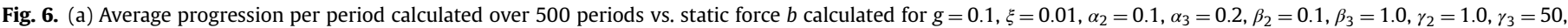
$\varphi=0, \omega=0.35$. (b) Typical bifurcation diagram showing the dependence of the drill-bit velocity on the static force for $a=0.5$ and the same parameters as in part (a).
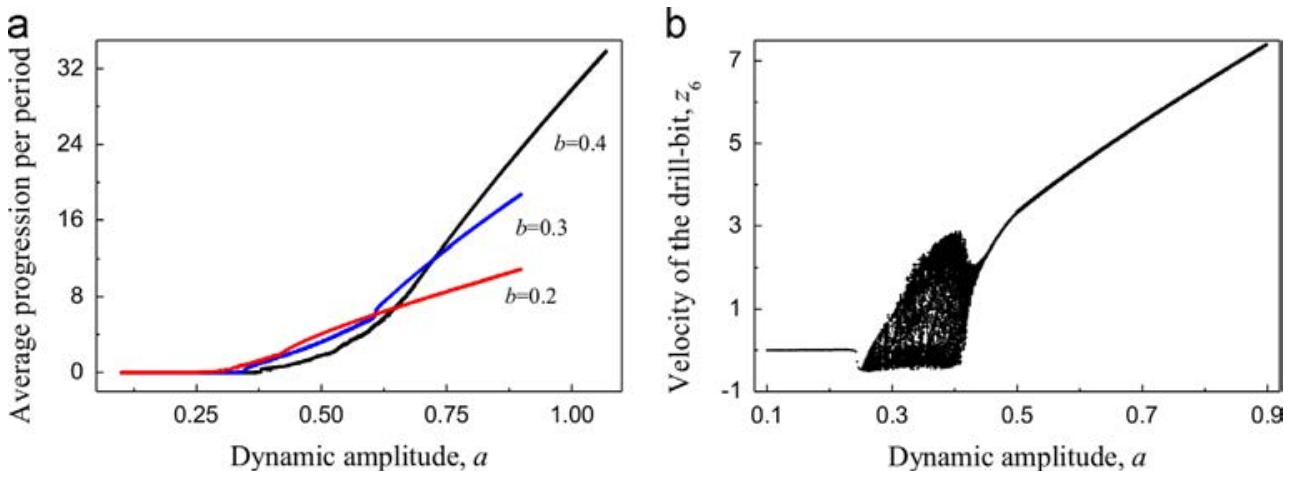

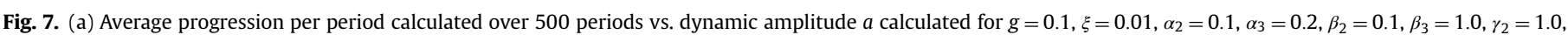

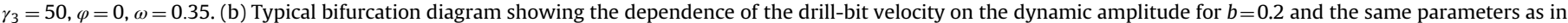
part (a). 

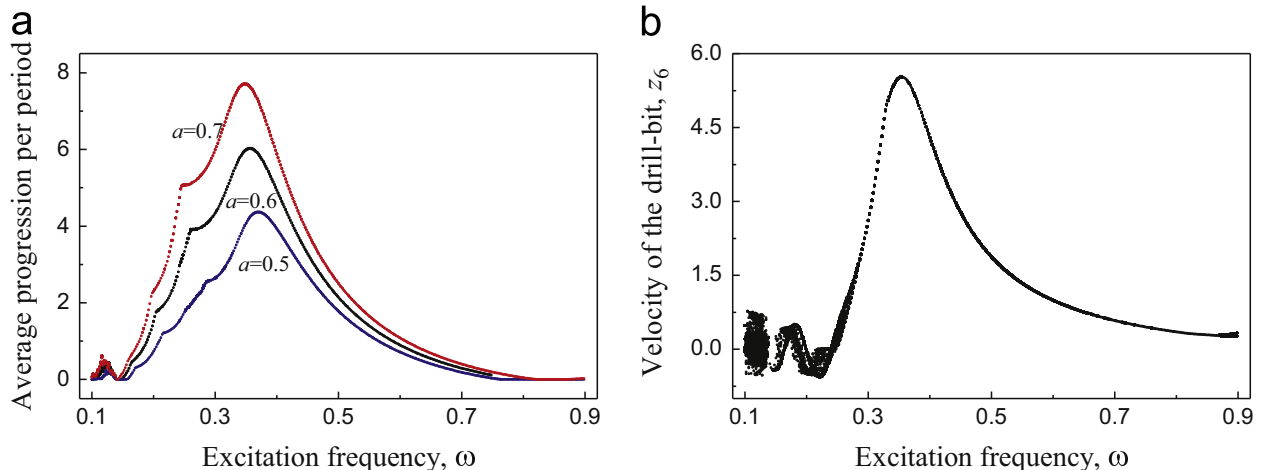

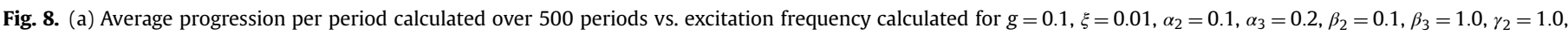

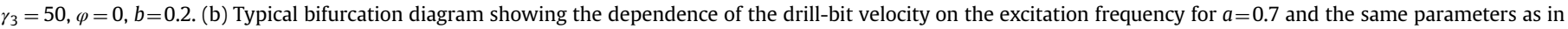
part (a).

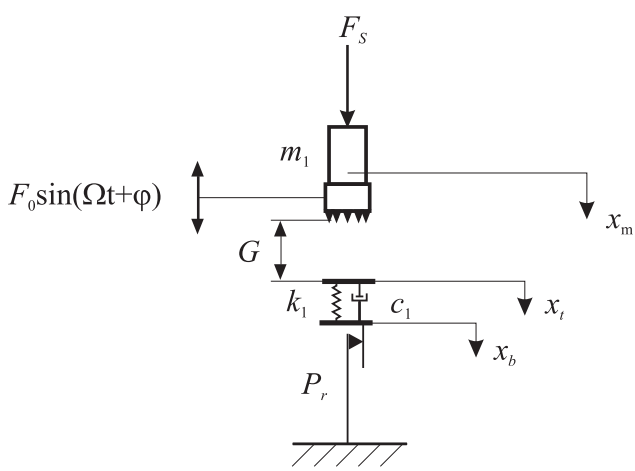

Fig. 9. Low dimensional model [22] used to describe the interactions between the drill-bit and the rock.

values of the static force, $b$. It is surprising to note that in the range $0.3<a<0.6$, the lower static force $b=0.2$ delivers the highest progression. Our analysis shows that in the part of this range $0.42<a<0.60$ the system responds periodically for $b=0.2$ (see Fig. 7(b)) and it operates within the optimal dynamic amplitude range. In contrast, for $b=0.3,0.4$, the system response is chaotic and therefore less effective from the progression point of view. Our simulations also show that chaotic regimes are observed for $a<0.6$ for $b=0.3$ and for $a<0.73$ for $b=0.4$. It is evident from the presented results that it is more effective to operate with a smaller static force in the periodic regime than with a large static force in the chaotic regime.

\subsection{Influence of excitation frequency}

Excitation frequency is the most important factor governing the progression of the drill-bit. To study the effect of a change in excitation frequency on progression, the RED model was simulated for varying excitation amplitudes giving the results shown in Fig. 8(a). As can be seen from this figure, for each curve corresponding to different dynamic amplitudes there is a well pronounced maximum in average progression per period. It is located within the periodic range of system response as shown in a typical bifurcation diagram in Fig. 8(b). It is important to note that maxima for all considered dynamic amplitudes lie in the frequency range $0.3<\omega<0.4$. If the frequency increases further, the system response remains periodic but the rate of progression drops. This result for the higher dimensional system corresponds well with the previous conclusions obtained for the simple one dimensional model.

\section{Comparison with the low-dimensional model [22]}

One of the simplest physical models which could be used to describe the percussive drilling was proposed in [22] and it is composed of a mass loaded by a force having static and harmonic components, and a dry friction slider, as shown in Fig. 9. This model has been extensively studied in the past $[23,24]$ and some key findings are briefly summarised below. Then a comparison with the results described above is carried out.

The following non-dimensional variables and parameters were used in the calculations:

$\Omega_{0}=\sqrt{\frac{k_{1}}{m_{1}}}, \quad \omega=\frac{\Omega}{\Omega_{0}}, \quad a=\frac{F_{0}}{P_{r}}, \quad b=\frac{F_{s}}{P_{r}}$,

$\xi=\frac{c_{1}}{2 m_{1} \Omega_{0}}, \quad g=\frac{k}{P_{r}} G$

$\tau=\Omega_{0} t, \quad z_{1}=x_{m} \frac{k_{1}}{P_{r}}, \quad z_{2}=\frac{d z_{1}}{d \tau}$,

$z_{3}=x_{t} \frac{k_{1}}{P_{r}}, \quad z_{4}=x_{b} \frac{k_{1}}{P_{r}}$.

The same three regimes of operation exist in this low dimensional model and they are described by the equations given below:

$z_{1}^{\prime}=z_{2}$

$z_{2}^{\prime}=a \cos (\omega \tau+\phi)+b-\mathcal{P}_{1} \mathcal{P}_{2}\left(1-\mathcal{P}_{3}\right)\left(2 \xi z_{2}+z_{3}-z_{4}\right)-\mathcal{P}_{1} \mathcal{P}_{3}$,

$z_{3}^{\prime}=\mathcal{P}_{1} z_{2}-\left(1-\mathcal{P}_{1}\right)\left(z_{3}-z_{4}\right) /(2 \xi)$,

$z_{8}^{\prime}=\mathcal{P}_{1} \mathcal{P}_{2} \mathcal{P}_{3}\left(\left(z_{7}-z_{8}-1\right) /(2 \xi)+z_{6}\right)$,

where $\mathcal{P}_{1}=H\left(z_{3}-z_{4}\right), \mathcal{P}_{2}=H\left(2 \xi z_{2}+z_{3}\right), \mathcal{P}_{3}=H\left(2 \xi z_{2}+z_{3}-1\right)$ and $\mathcal{P}_{4}=H\left(z_{2}\right)$

Typical bifurcation diagrams showing the dependence of the average progression per period on the applied static force are shown in Fig. 10(a). Here four different curves correspond to different levels of the dynamic amplitude, $a=0.25,0.35,0.45$ and 0.55 and all other parameters are specified in the caption of this figure. As can be seen from the velocity bifurcation diagram shown in Fig. 10(b) for $a=0.35$, the response of the system is typically chaotic for the low values of the static force and in general the average progression per period is low in this region. The best progression is achieved when the system response is periodic and the frequency of the response is the same as the frequency of the applied dynamic force. For the lower values of the dynamic amplitudes, applying a higher static force will completely suppress the progression resulting in zero penetration rate until the static force becomes large enough to break the rock even with very little contribution of the dynamic force (see for example curve for $a=0.35$ ) 

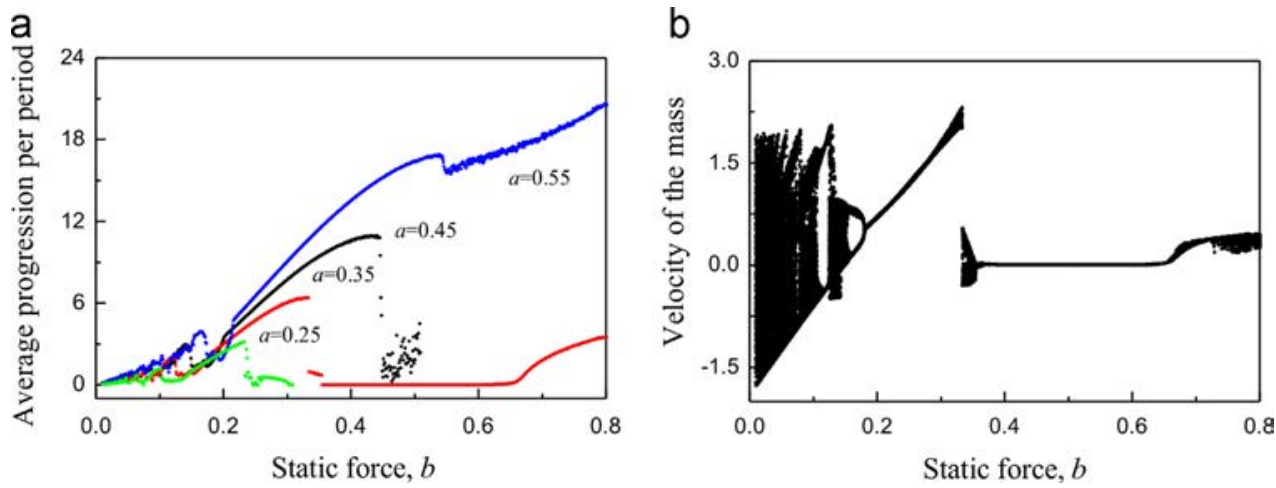

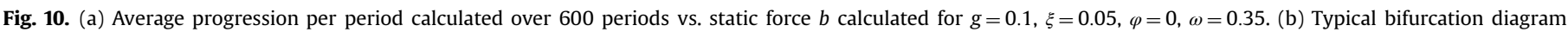
showing the dependence of the drill-bit velocity on the static force for $a=0.35$ and the same parameters as in part (a).
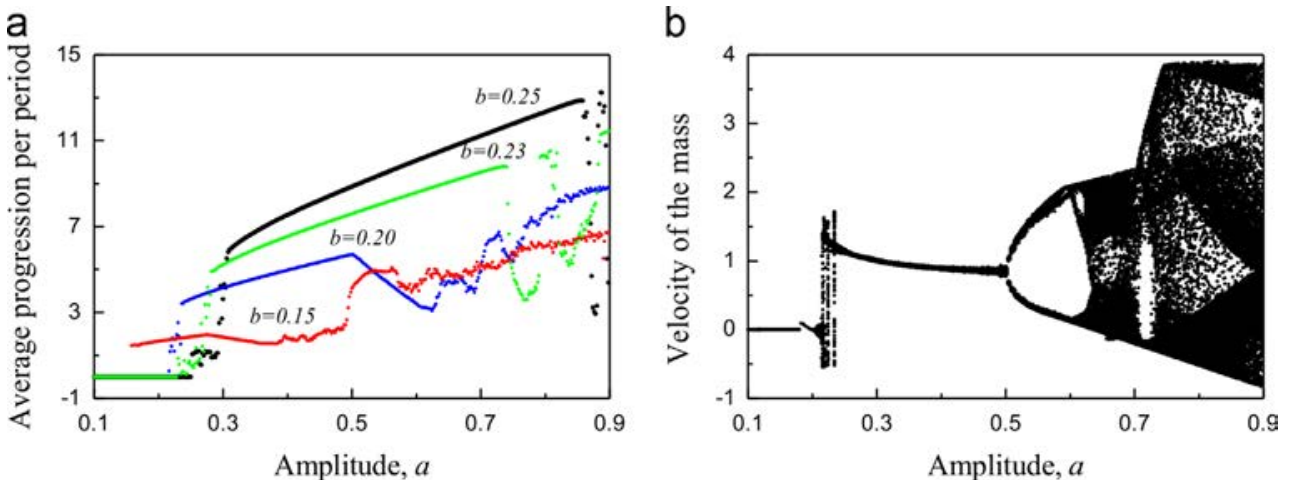

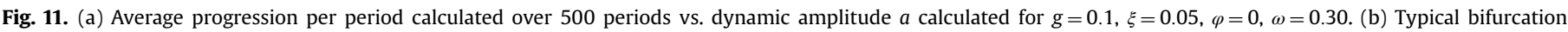
diagram showing the dependence of the drill-bit velocity on the dynamic amplitude for $b=0.2$ and the same parameters as in part (a).

It is important to note here that the progression rates predicted by this simple model in general are much higher than the rates discussed in Section 3. This is not surprising as the mass of the whole system described in the previous section is much higher and the same level of the static force applied would not produce the same progression speed for these two different systems. However the general trend and the strategy of choosing the optimal static force to achieve the best progression remain the same for these two models - the static force has to be chosen to ensure that the system response is periodic with the frequency of the applied force and the value of the static force is smaller than the amplitude of the applied dynamic excitation.

The dependence of the system response on the dynamic amplitude is shown in Fig. 11 for a number of values of the static force, $b=0.15,0.20,0.23$ and 0.25 . As can be seen from Fig. 11(a), the progression rates are equal to zero for very low dynamic amplitudes where the combined effects of static and dynamic forces is not strong enough to break the rocks and move the system forwards. As the dynamic amplitude increases, the average progression per period also increases and again the optimal drilling regimes are achieved when the system response is periodic. Analysing the system responses and the corresponding progression rates, we note that for the lower values of the static force, the rates of penetration drop after the period doubling bifurcation as observed for $a \approx 0.5$ and $b=0.2$ in Fig. 11(b).

In general the low dimensional model predicts similar trends in system behaviour as does the larger model introduced in Section 3. Specifically it also forecasts zero progression rates for lower amplitudes and significant improvements of the average progression rates with increasing amplitude. The period-1 system response is again observed as the most beneficial from the progression point of view and any bifurcations to period-2 or chaotic responses should be avoided during operation to maintain high drilling rates. It should be noted that the period doubling bifurcations and the chaotic behaviour at the higher amplitude values are observed for the low dimensional model only. However despite the differences in the two model predictions at the higher amplitude values, the strategy of choosing the optimal amplitude remains the same in both cases.

Finally, the influence of the frequency of the applied force on the system response is shown in Fig. 12. Here four curves are presented for different values of the dynamic amplitude, $a=0.35$, $0.40,0.45$ and 0.55 , and constant value of the static force, $b=0.25$. As can be seen from part (b), for lower values of the dynamic amplitudes, the chaotic response is observed for lower frequencies. As the frequency increases, the periodic response is recorded and the average progression per period is decreasing with the increase of the frequency.

Comparing the results presented in Figs. 8 and 12, we note that the low dimensional model predictions do not coincide with the previous finding where the clear resonant frequency was identified for the considered frequency range for the specified sets of system parameters. The progression rates are also much higher for the lower dimensional model. However the similar results were recorded at the higher frequencies where the progression rates drop to zero according to both models. The significant differences between two models at the lower frequencies could be explained by two factors. Firstly, internal resonances and interplay between the natural frequencies of the three masses model from Section 3 affect the global system response at different excitation frequencies and in the result produce nonlinear resonance shown in Section 3. Secondly, as was discussed above, the same static force applied to the much heavier mass will produce smaller progression rates and hence it explains the differences in the level of the average progression achieved. 

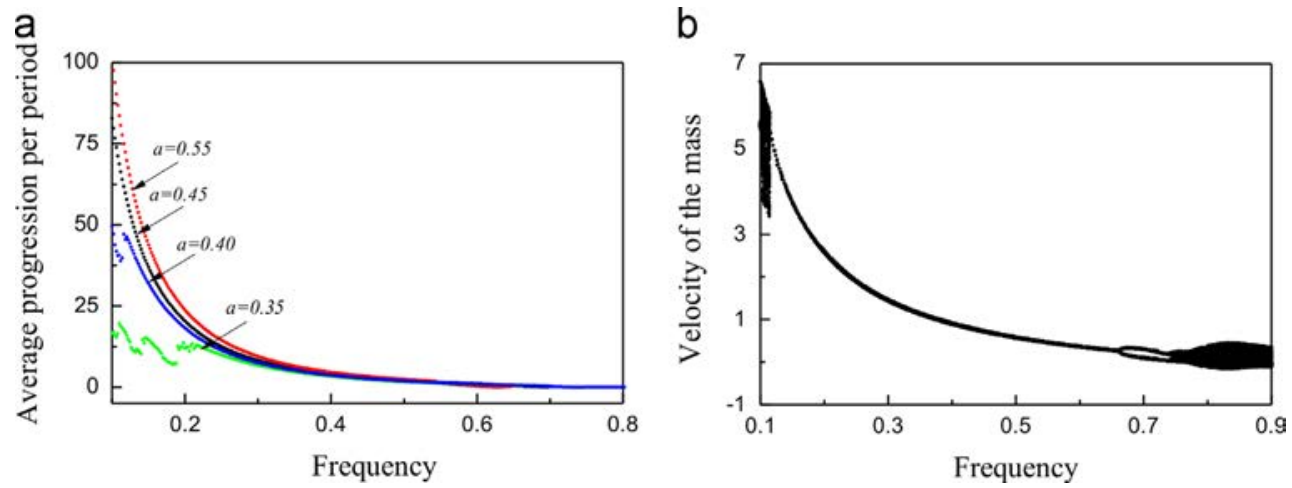

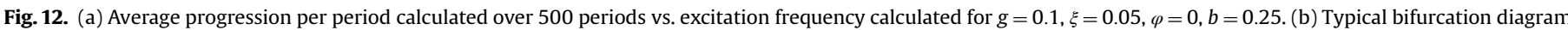
showing the dependence of the drill-bit velocity on the excitation frequency for $a=0.4$ and the same parameters as in part (a).

\section{Conclusions}

This paper presents the modelling of the vibro-impact drilling system showing the results of the numerical analysis and comparison between two models. The first one is a newly developed model of an existing experimental rig (three masses model) and the second one is a simplified low dimensional model [22] created to describe the dynamic interaction between the drill-bit and the drilled formation. Based on the analysis of the system responses, the optimal loading parameters were identified and the influence of the additional degrees-of-freedom on the loading optimisation strategy was investigated.

Three main control parameters were considered, namely the applied static force, and the amplitude and the frequency of the applied dynamics force. According to both models the best progression rates are achieved when the system response is periodic and the frequency of the response is the same as the frequency of the applied dynamic force (i.e. period- 1 response is observed), and the value of the static force is smaller than the amplitude of the applied dynamic excitation. In both cases, zero progression rates were obtained for lower values of the excitation amplitudes and the average progression increased with the increase in the dynamic amplitudes. Both models also predict zero progression rates at the higher excitation frequencies.

According to the higher dimensional model simulations, the optimal static force can be found for the chosen value of the excitation amplitude and for small and medium amplitudes it can be estimated as $40-45 \%$ of the amplitude value. The appropriate choice of the excitation frequency is very important, and for the higher dimensional model the optimal non-dimensional frequency range of the drilling system is $0.3<\omega<0.5$. Below the lower boundary the system response is chaotic and above the upper boundary progression rates decrease as the frequency increases.

Finally, it can be concluded that low dimensional model provides good estimates of the optimal static force and the amplitude of the dynamic force, and it could be used for the operational control of the drilling system to adjust the loading parameters while drilling through different formations. The choice of the optimal frequency, however, should be made based on the predictions of the more detailed model of the drilling system as additional degrees of freedom significantly influence the structure of the internal resonances and they should be taken into account.

\section{References}

[1] Samuel GR. Percussion drilling. Is it a lost technique? A review. In: SPE 35240, The Permian basin oil \& gas recovery conference, Mildland, TX, USA, 1996.

[2] Rabia H. A unified prediction model for percussive and rotary drilling. Mining Science and Technology 1985;2(3):207-16.
[3] Guarin PL, Arnold HE, Harpst WE, Davis EE. Rotary percussion drilling. In: AP 49-112, Twenty nine annual meeting, Chicago, November 1949.

[4] Topanelian E. Effect of low frequency percussion in drilling hard rock. AIMEPetroleum Transaction 1958;213.

[5] Bates RE. Field results of percussion air drilling. In: SPE 886, The 39th SPE annual fall meeting Houston, TX, USA, October 1964

[6] Smith FW, Kopczynksi W. Oilfield percussion drilling. In: SPE 222, The 32nd annual California regional meeting of SPE, Bakerfield, CA, USA, 1961.

[7] Simon R. Energy balance in rock drilling. Society of Petroleum Engineers Journal 1963:298-306; Transactions of the AIME 228.

[8] Simon R. Transfer of stress wave energy in the drill steel of a percussive drill to the rock. Journal of Rock Mechanics and Mining Science \& Geomechanics Abstracts 1964;1(3):397-411.

[9] Hustrulid WA, Fairhurst C. A theoretical and experimental study of the percussive drilling of rock. Part I-theory of percussive drilling. International Journal of Rock Mechanics \& Mining Science and Geomechanics Abstracts 1971:8(4):311-33.

[10] Hustrulid WA, Fairhurst C. A theoretical and experimental study of the percussive drilling of rock. Part II-force-penetration and specific energy determinations. International Journal of Rock Mechanics \& Mining Science and Geomechanics Abstracts 1971;8(4):335-40.

[11] Hustrulid WA, Fairhurst C. A theoretical and experimental study of the percussive drilling of rock. Part III-experimental verification of the mathematical theory. International Journal of Rock Mechanics \& Mining Science and Geomechanics Abstracts 1972;9(3):417-29.

[12] Hustrulid WA, Fairhurst C. A theoretical and experimental study of the percussive drilling of rock. Part IV-application of the model to actual percussion drilling. International Journal of Rock Mechanics \& Mining Science and Geomechanics Abstracts 1972;9(3):431-42.

[13] Lundberg B. Energy transfer in percussive rock destruction-I: comparison of percussive methods. International Journal of Rock Mechanics \& Mining Science and Geomechanics Abstracts 1973:10(5):381-99.

[14] Lundberg B. Energy transfer in percussive rock destruction-II: supplement on hammer drilling. International Journal of Rock Mechanics \& Mining Science and Geomechanics Abstracts 1973:10(5):401-41.

[15] Lundberg B. Energy transfer in percussive rock destruction-III: stress wave transmission through joints. International Journal of Rock Mechanics \& Mining Science and Geomechanics Abstracts 1973:10(5):421-35.

[16] Lundberg B, Okrouhlik M. Influence of 3D effects on the efficiency of percussive rock drilling. International Journal of Impact Engineering 2001:25:345-60.

[17] Lundberg B, Okrouhlik M. Efficiency of a percussive rock drilling process with consideration of wave energy radiation into the rock. International Journal of Impact Engineering 2006;32(10):1573-83.

[18] Franca LFP, Weber HI. Experimental and numerical study of a new resonance hammer drilling model with drift. Chaos, Solitons \& Fractals 2004;21 (4):789-801.

[19] Franca LFP. A bit-rock interaction model for rotary-percussive drilling. International Journal of Rock Mechanics and Mining Sciences 2011;48(5):827-35.

[20] Krivtsov AM, Wiercigroch M. Dry friction model of percussive drilling. Meccanica 1999:34:425-34.

[21] Wiercigroch M, Wojewoda J, Krivtsov AM. Dynamics of ultrasonic percussive drilling of hard rocks. Journal of Sound and Vibration 2005;280:739-57.

[22] Pavlovskaia E, Wiercigroch M, Grebogi C. Modeling of an impact system with a drift. Physical Review E 2001;64:056224.

[23] Pavlovskaia E, Wiercigroch M. Analytical drift reconstruction for visco-elastic impact oscillators operating in periodic and chaotic regimes. Chaos, Solitons \& Fractals 2004;19:151-61.

24] Pavlovskaia E, Wiercigroch M. Low dimensional maps for piecewise smooth oscillators. Journal of Sound and Vibration 2007:305:750-71.

[25] Ajibose OK, Wiercigroch M, Pavlovskaia E, Akisanya AR. Global and local dynamics of drifting oscillator for different contact force models. International Journal of Non-Linear Mechanics 2010;45:850-8. 
[26] Ajibose OK, Wiercigroch M, Karolyi Gy, Pavlovskaia EE, Akisanya AR. Dynamics of the drifting impact oscillator with new model of the progression phase. Journal of Applied Mechanics 2012;79:061007 9 pp.

[27] Han G, Bruno M, Lao K. Percussion drilling in oil industry: review and rock failure modeling. In: AADE-05-NTCE-59, 2005. AADE National technical conference \& exhibition, Houston, TX, April 2005.

[28] Wiercigroch M. Resonance enhanced drilling: method and apparatus. Patent No. WO2007141550, 2007

[29] Pavlovskaia E, Wiercigroch M. Periodic solutions finder for vibro-impact oscillator with a drift. Journal of Sound and Vibration 2003;267:893-911.
[30] Pavlovskaia EE, Wiercigroch M, Woo K-C, Rodger AA. Modelling of ground moling dynamics by an impact oscillator with a frictional slider. Meccanica 2003;38:85-97.

[31] Wiercigroch M, Pavlovskaia EE. Nonlinear dynamics of vibro-impact systems: 49 theory and experiments. In: Cartmell M, editor. Proceedings of the 5th international conference on modern practice 50 in stress and vibration analysis, September 9-11, 2003, Glasgow, 2003. p. 513-20. 